\title{
Worldwide harmonization of emission regulations - a dream never becomes reality?
}

Dr. Manfred Schuckert, Daimler AG

Zum Zeitpunkt der Drucklegung lagen diese Unterlagen noch nicht vor. Wir bitten um Verständnis. 